\title{
TOPOLOGICAL PHASE TRANSITIONS IN THE NONLINEAR PARALLEL ISING MODEL*
}

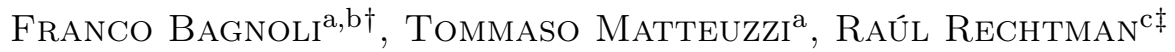 \\ ${ }^{a}$ Dipartimento di Fisica e Astronomia, Università di Firenze \\ Via G. Sansone 1, 50019 Sesto Fiorentino, Italy \\ ${ }^{\mathrm{b}} \mathrm{CSDC}$ and INFN, Sez. Firenze, Italy \\ ${ }^{\mathrm{c}}$ Instituto de Energías Renovables, Universidad Nacional Autónoma de México \\ Apdo. Postal 34, 62580 Temixco, Mor., Mexico
}

\section{(Received February 29, 2016)}

We investigate some phase transitions of a nonlinear, parallel version of the Ising model, characterized by an antiferromagnetic linear coupling and a ferromagnetic nonlinear one. This model arises in problems of opinion formation. The mean-field approximation shows chaotic oscillations by changing the linear coupling or the connectivity. The spatial model exhibits bifurcations in the average magnetization, similar to what is seen in the mean-field approximation induced by the change of the topology, after rewiring short-range to long-range connections as predicted by the small-world effect. These coherent periodic and chaotic oscillations of the magnetization reflect a certain degree of synchronization of the spins, induced by long-range couplings. Similar bifurcations may be induced in the randomly connected model by changing the coupling or the connectivity and the synchronism of the updating (dilution of the rule).

DOI:10.5506/APhysPolBSupp.9.37

\section{Introduction}

Models of opinion formation are often based on some version of the Ising model [1], where conformists are modelled by a ferromagnetic coupling and contrarians by an antiferromagnetic one.

One of the most intriguing effects is the hipster's one, in which a society of contrarians tends to behave in a uniform way [2]. Clearly, "conformistic hipsters" always change their behaviour, when they realize to be still in the

\footnotetext{
* Presented at the Summer Solstice 2015 International Conference on Discrete Models of Complex Systems, Toronto, Ontario, Canada, June 17-19, 2015.

$\dagger$ franco.bagnoli@unifi.it

‡ rrs@ier.unam.mx
} 
mainstream, but since that do so all together, they remain synchronized. This periodic synchronization is similar to that of pendula and can happen also in continuous systems.

Individuals that are under a strong social pressure tend to agree with the great majority even when they are certain that the majority's opinion is wrong, as shown by Asch [3].

An agent in our model can be either a conformist, that agrees with his neighbours, or a contrarian that disagrees. If the total effect is just the sum of individual terms, we say that this is a linear interaction. In order to include the observations by Asch, we establish that even contrarian agents, under a strong social pressure, tend to agree with the majority. Since this effect manifests itself only when the majority is near total consensus, we say that it is due to nonlinear interactions (the effect does not grow linearly with the number of neighbours holding a given opinion).

The strategy of following an overwhelming majority is reasonable in a competitive environment like trading markets, since it is probable that this coherent behaviour is due to some unknown piece of information, and in any case, the competitive loss is minimal since it equally affects all other agents.

In previous works $[4,5]$, we studied the "nonlinear" behaviour of contrarians, where the effect of overwhelming majority was present only above a given threshold, denoting them with the term "reasonable contrarian". We investigated the collective behaviour of a society composed either of reasonable contrarian agents, or a mixture of contrarians and conformists. The rationale was that in some cases, and, in particular, in the presence of frustrated situations like in minority games [6], it is not convenient to always follow the majority, since in this case, one is always on the "losing side" of the market. This is one of the main reasons for the emergence of a contrarian attitude. On the other hand, if all or almost all agents in a market take the same decision, it is often wise to follow such a trend. We can denote such a situation with the term "social norm".

A society composed of a strong majority of reasonable contrarians exhibits interesting behaviours when changing the topology of the connections. On a one-dimensional regular lattice, there is no long-range order, the evolution is disordered and the average opinion is always halfway between the extreme values. However, adding long-range connections or rewiring existing ones, we observe the Watts-Strogatz "small-world" effect, with a transition towards a mean-field behaviour. But since in this case the mean-field equation is, for a suitable choice of parameters, chaotic, we observe the emergence of coherent oscillations, with a bifurcation cascade eventually leading to a chaotic-like behaviour of the average opinion. The small-world transition is essentially a synchronization effect. Similar effects with a bifurcation 
diagram resembling that of the logistic map have been observed in a different model of "adapt if novel - drop if ubiquitous" behaviour, upon changing the connectivity $[7,8]$.

The goal of the present study is that of reformulating this problem in terms of a parallel, nonlinear Ising model. This paper is related to the one by Bagnoli, Matteuzzi and Rechtman in this same issue [9].

\section{The nonlinear model}

We consider a system with $N$ sites, each one in a state $s_{i} \in\{-1,1\}$, $i=0, \ldots, N-1$. The state of the system is $s=\left(s_{0}, \ldots, s_{N-1}\right)$. The topology

of the system is defined by the adjacency matrix $a$ with $a_{i j}=1$ if site $j$ belongs to site $i$ s neighbourhood and is zero otherwise. The connectivity $k_{i}$ and the local field $h_{i}$ of site $i$ are

$$
k_{i}=\sum_{j} a_{i j}, \quad \tilde{h}_{i}=\sum_{j} a_{i j} s_{j}, \quad h_{i}=\frac{\tilde{h}_{i}}{k_{i}} .
$$

It is clear that $h_{i} \in[-1,1]$. The magnetization $m$ is defined by

$$
m=\frac{1}{N} \sum_{i} s_{i}
$$

with $m \in[-1,1]$.

The Hamiltonian of the model is

$$
\mathcal{H}(s)=-J \sum_{i} h_{i} s_{i}
$$

The dynamics of the model (i.e., Monte Carlo simulations) is given here by the heat-bath transition probabilities

$$
\tau\left(s_{i}^{\prime} \mid h_{i}\right)=\frac{1}{1+\exp \left(-2 J s_{i}^{\prime} h_{i}\right)}=\frac{1}{2}\left[1+\tanh \left(J s_{i}^{\prime} h_{i}\right)\right]
$$

where $s_{i} \equiv s_{i}(t)$ and $s_{i}^{\prime} \equiv s_{i}(t+1)$ and the coupling terms have been rescaled with the temperature. The dynamical model can be updated in a sequential or parallel order; the sequential dynamics brings to the equilibrium distribution (in the case of symmetric couplings), while the fully parallel version has a different equilibrium distribution [10], although many observables like the magnetization take the same value $[9,11]$. 
Let us consider multi-spin interactions, for instance up to four spins as depicted in figure 1. The Hamiltonian of the model becomes

$\mathcal{H}(s)=-\sum_{i} s_{i}\left(H+J \sum_{j} a_{i j} s_{j}+Z \sum_{j k} a_{i j} a_{j k} s_{j} s_{k}+W \sum_{j k l} a_{i j} a_{i k} a_{i l} s_{j} s_{k} s_{l}\right)$.

It is possible to recast the interactions in terms of the local field $h_{i}$ only. For simplifying the notation, we denote with $s^{\prime}=s_{i}$ the updating spin, and with $s_{1}, s_{2}, s_{3}$ the three connected neighbours (i.e., those for which $a_{i j} \neq 0$, regardless of their spatial relationship). Considering only the terms containing $s^{\prime}$ (the others simplifies in the transition probabilities), we have

$$
\begin{aligned}
& 2-\operatorname{spin}: s^{\prime}\left(s_{1}+s_{2}+s_{3}\right) \\
& 3-\operatorname{spin}: s^{\prime}\left(s_{1} s_{2}+s_{1} s_{3}+s_{2} s_{3}\right) \\
& 4-\operatorname{spin}: s^{\prime}\left(s_{1} s_{2} s_{3}\right)
\end{aligned}
$$

and considering powers of the local field $\tilde{h}$ (not rescaled here with the connectivity),

$$
\begin{aligned}
\tilde{h} & =s_{1}+s_{2}+s_{3} ; \\
\tilde{h}^{2} & =\left(s_{1}+s_{2}+s_{3}\right)^{2}=3+2\left(s_{1} s_{2}+s_{1} s_{3}+s_{2} s_{3}\right) ; \\
\tilde{h}^{3} & =\left(s_{1}+s_{2}+s_{3}\right)^{3}=7 \tilde{h}+6 s_{1} s_{2} s_{3}
\end{aligned}
$$

we see that, by rescaling the interaction terms to include the connectivity and the constant factors, one can obtain all plaquette contributions.

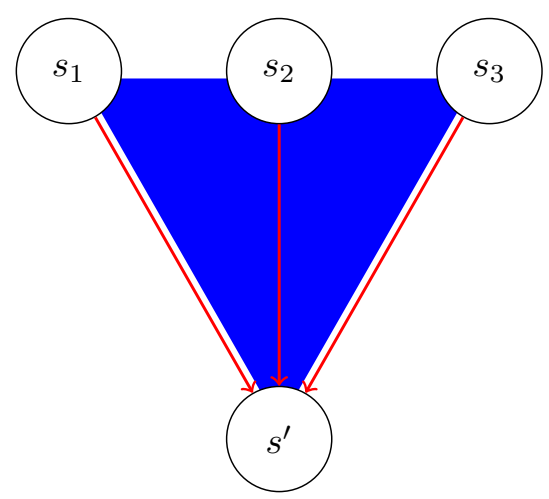

Fig. 1. The plaquette terms in the one-dimensional model.

In the following, we shall consider a pair $(h)$ and four-spin $\left(h^{3}\right)$ terms in order to keep the system symmetric with respect to spin inversion (as the linear version). Here, the nonlinearity is related to the powers of local field. 
The local transition probability is thus given by

$$
\tau\left(s_{i}^{\prime} \mid h_{i}\right)=\frac{1}{1+\exp \left(-2 s_{i}^{\prime}\left(J h_{i}+W h_{i}^{3}\right)\right)}=\frac{1}{2}\left\{1+\tanh \left[s_{i}^{\prime}\left(J h_{i}+W h_{i}^{3}\right)\right]\right\} .
$$

The term $J$ models the "linear" effects of neighbours, so $J>0$ gives a conformistic (ferromagnetic) behaviour and $J<0$ a contrarian (antiferromagnetic) one. The term $W$ models the nonlinear effects of the crowd. One can model the effects of an overwhelming majority (Asch) by inserting $J<0$ (contrarian attitude) and $W>0$ (social norms).

\subsection{Mean-field approximation}

The Markov equation for the probability distribution of the configuration $s$ in the fully parallel case is

$$
P\left(\boldsymbol{s}^{\prime}, t+1\right)=\sum_{\boldsymbol{s}}\left[\prod_{i=1}^{N} \tau\left(s_{i}^{\prime} \mid \boldsymbol{s}\right)\right] P(\boldsymbol{s}, t) .
$$

The simplest mean-field approximation is built discarding spatial correlations among spins. In this case, the joint probability distribution factorizes $P(s, t)=\prod_{i} \pi\left(s_{i}, t\right)$ and we get, for a generic spin and considering a fixed connectivity $K$,

$$
\pi\left(s^{\prime}, t+1\right)=\sum_{s_{1}, s_{2}, \ldots, s_{K}} \tau\left(s^{\prime} \mid h\right)\left[\prod_{i} \pi\left(s_{i}, t\right)\right],
$$

where $h=\left(s_{1}+s_{2}+\cdots+s_{K}\right) / K=\tilde{h} / K$ and the subscripts now identify the spins connected with $s^{\prime}$.

We can introduce the mean value of the magnetization $m \equiv m(t)=$ $2 \pi(1, t)-1$ and since the transition rule depends only on the sum $\tilde{h}$ of the spin values, we get

$$
\begin{aligned}
m^{\prime}= & \frac{1}{2^{K}} \sum_{\tilde{h}=0}^{K}\left(\begin{array}{c}
K \\
\tilde{h}
\end{array}\right)(1+m)^{\tilde{h}}(1-m)^{\tilde{h}-K} \\
& \times \tanh \left[J\left(2 \frac{\tilde{h}}{K}-1\right)+W\left(2 \frac{\tilde{h}}{K}-1\right)^{3}\right],
\end{aligned}
$$

where, as usual, $m^{\prime}=m(t+1)$, see Fig. $2(\mathrm{~b})$.

The mean-field return map exhibits chaotic phases and bifurcations: period-doubling and pitchfork ones. The pitchfork bifurcations are due to the symmetry of the map, Fig. 2(b): one can have disjoint symmetric 


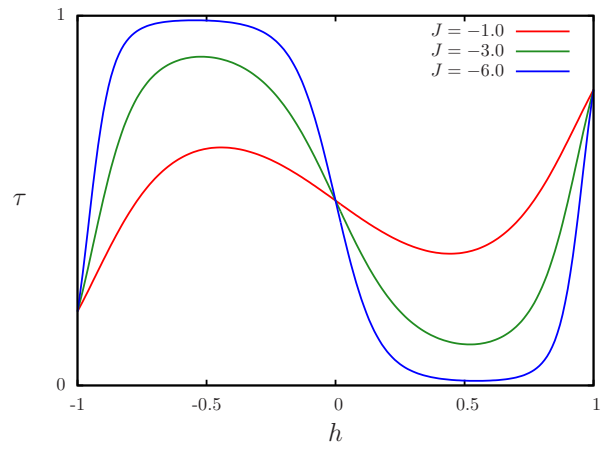

(a)

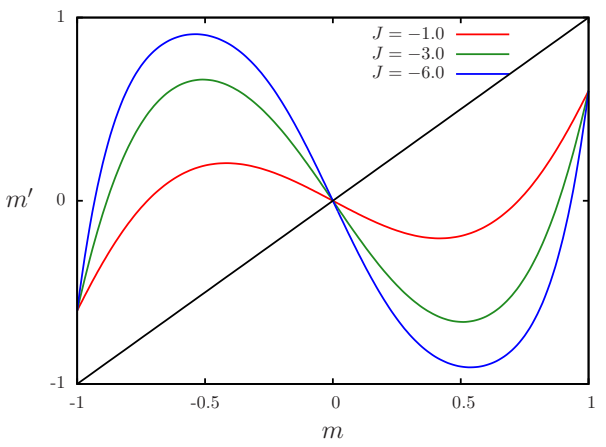

(b)

Fig. 2. (a) Transition probabilities and (b) mean-field map, Eq. (2) for the nonlinear Ising model, for $J=-1, W=2.693 ; J=-3, W=3.693 ; J=-6, W=6.693$ (the values of $W$ have been chosen in order to have all curves meet for $h= \pm 1$ ).

attractors that finally merge. The numerical bifurcation diagrams as function of $J$ and $K$ are shown in Fig. 3 (a) and (b), respectively. In order to cover all branches of the pitchfork bifurcations, we used several initial conditions for the magnetization $m$. These bifurcation diagrams are qualitatively very similar to the ones reported in Ref. [4]. This scenario is reminiscent of that of the logistic map (where, however, the pitchfork bifurcations are not present).

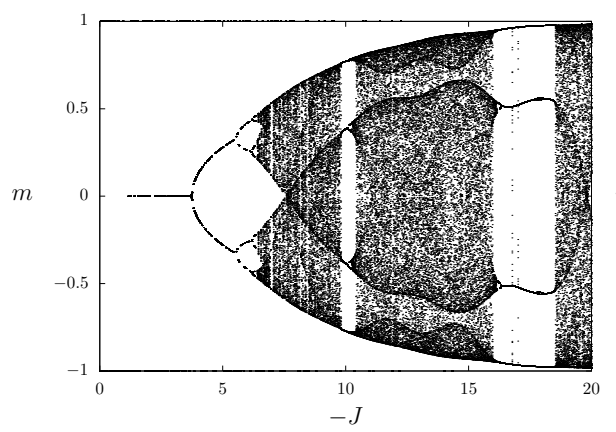

(a)

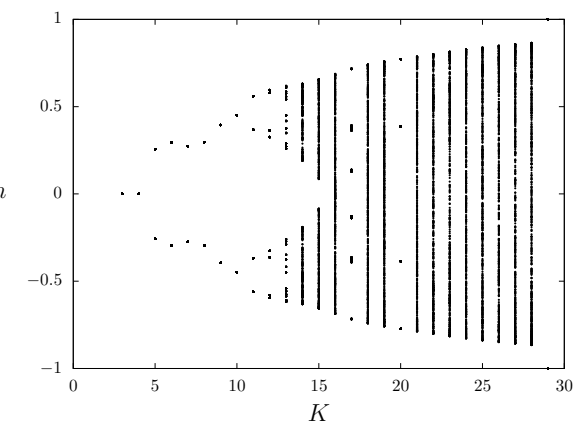

(b)

Fig. 3. Mean-field bifurcation diagram of the nonlinear parallel Ising model, (a) as a function of $J(K=20)$ and (b) as a function of $K(J=10)$. Here, $W=20,200$ iterations after a transient of 1000 steps, three initial conditions $\left(m_{0}=0.2,0.5,0.8\right)$.

\subsection{Topological phase transitions}

The fully parallel version defines a probabilistic, totalistic cellular automaton, whose transition probabilities are given by Eq. (1), applied to all sites. The behaviour of the one-dimensional model with a finite number of 
interactions is not very interesting, since in this configuration and in the absence of absorbing states, one cannot have true phase transitions (this situation corresponds, for the randomly connected case, to the dilution parameter $d=1$, see Section 3 and Fig. 7).

In order to recover the mean-field behaviour and to be more adherent to reality, we rewired a fraction $p$ of links to random sites, as in the scheme by Watts and Strogatz [12]. Notice that in this case, the interactions are no more symmetric.

The numerical simulations exhibit topologically induced bifurcations, as shown in Fig. 4. We can observe transitions as a function of $p$, Fig. 4, as well as a function of $J$, Fig. 5 (a), and $K$, Fig. 5 (b), shown for $p=1$.

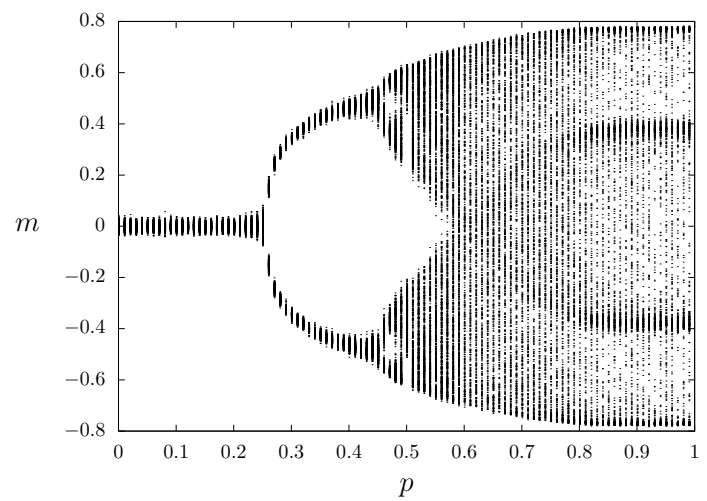

Fig. 4. Bifurcations as a function of long-range rewiring probability $p$. Here, $J=$ $-10, W=20 p=1,500$ points, $N=40,000$, transient of 2000 steps, two initial conditions $\left(m_{0}=0.2,0.8\right)$. The gap near $p=0.5$ is a pitchfork bifurcation with a branch not captured by the initial magnetization.

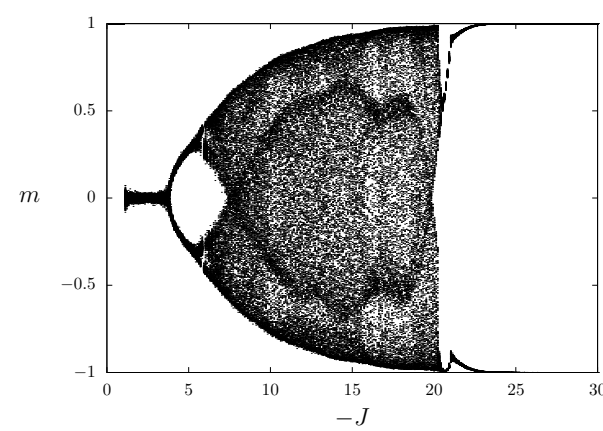

(a)

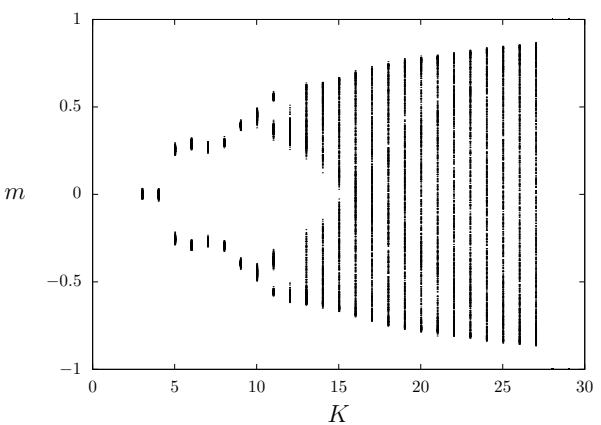

(b)

Fig. 5. (a) Bifurcations as a function of $p$ and (b) as a function of $K$. Here, $J=-10, W=20, p=1,400$ points, $N=10,000$, transient of 1000 steps, two initial conditions $\left(m_{0}=0.2,0.8\right)$. 


\subsection{Mapping parameters}

There are some similarities between the bifurcation diagrams for $p=1$ as a function of $J$ and of $K$, Fig. 3, and, in general, among all bifurcation diagrams. Indeed, it can be shown that it is possible to obtain a mapping among parameters, i.e., the behaviour of the model for a certain value of $J$, $W$ and $K$ is the same if we increase these parameters in a proper way. We use a continuous approximation of the mean-field equation.

By using Stirling's approximation for the binomial coefficients in Eq. (2), for intermediate values of $c$ [13], we obtain

$$
\left(\begin{array}{c}
K \\
n
\end{array}\right) c^{n}(1-c)^{K-n} \simeq \frac{1}{\sqrt{2 \pi K c(1-c)}} \exp \left[\frac{-K(n / K-c)^{2}}{2 c(1-c)}\right]
$$

and therefore

$$
m^{\prime}=\int_{-\infty}^{\infty} d x \sqrt{\frac{K}{2 \pi\left(1-m^{2}\right)}} \exp \left(-\frac{K(x-m)^{2}}{2\left(1-m^{2}\right)}\right) \tau(1 \mid x) .
$$

By approximating $\tanh (x)$ with $x$, we can compute the convolution, obtaining (after reinserting the hyperbolic tangent)

$$
m^{\prime}=\tanh \left(\tilde{J} m+\tilde{W} m^{3}\right)
$$

with

$$
\left\{\begin{array}{l}
\tilde{J}=J+\frac{3 W}{K} \\
\tilde{W}=W\left(1-\frac{3}{K}\right) .
\end{array}\right.
$$

Notice that in the limit $K \rightarrow \infty, \tilde{J} \rightarrow J$ and $\tilde{W} \rightarrow W$.

The relation between parameters $J, K$ and $J_{1}, K_{1}$ of two mean-field approximations with different connectivities $K$ and $K_{1}$ is

$$
\left\{\begin{array}{l}
J_{1}=J+\frac{3}{K} W\left(1-\frac{K-3}{K_{1}-3}\right) \\
W_{1}=W \frac{K_{1}}{K} \frac{K-3}{K_{1}-3} .
\end{array}\right.
$$

The approximation of the hyperbolic tangent is valid for small $x$, so we expect that this scaling is better for large $K$, for which the convolution length is small. In Fig. 6, we report a mean-field map for four sets of corresponding values of the parameters. We see that the curves almost overlap. 


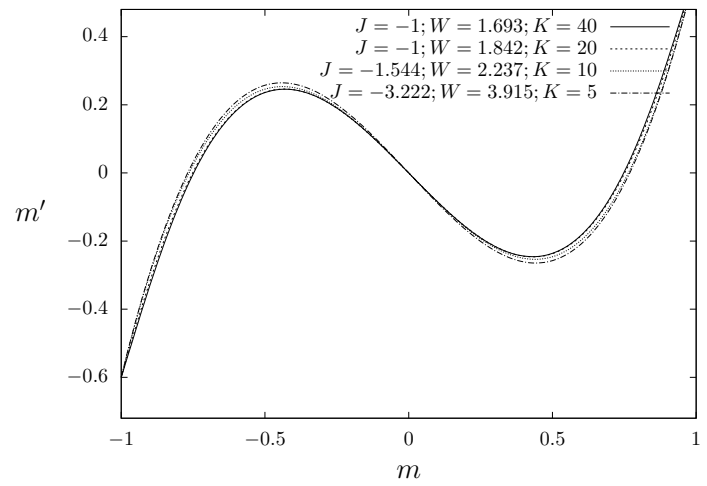

Fig. 6. Mean-field function, Eq. (2), for four set of corresponding parameters according with Eq. (5).

Since $J<0$ and $K>0$, the effect of this scaling is that of lowering the absolute value of $\tilde{J}$ and $\tilde{K}$ for small $K$ (larger than 3 ), so, given that for a large value of $K$ and certain values of $J$ and $W$, the mean-field equation is chaotic, it may be reduced to a fixed point graph by lowering the connectivity $K$.

\section{Partial asynchronism (dilution)}

We introduce the dilution $d$ in a way similar to what has been done in the sister article [9]. The dilution $d$ is the fraction of sites chosen at random that are not updated at every time step, i.e., a measure of the asynchronism of the updating rule.

We define the diluted rule as

$$
s_{i}(t+1)= \begin{cases}1 & \text { with probability }(1-d) \tau\left(h_{i}\right), \\ -1 & \text { with probability }(1-d)\left[1-\tau\left(h_{i}\right)\right] \\ s_{i}(t) & \text { otherwise, } i . e ., \text { with probability } d,\end{cases}
$$

so that for $d=0$ one has the standard parallel updating rule.

One time step is defined when, on the average, every site of the lattice is updated once. For a system with $N$ sites, the smallest value of the dilution is $d=1 / N$ and then $t_{d}=1 / d$ updates are needed to complete one time step. If $d=1 / 2, t_{d}=2$, etc.

As shown in figure 7 , also the dilution is able to trigger bifurcations in the spatial model. 


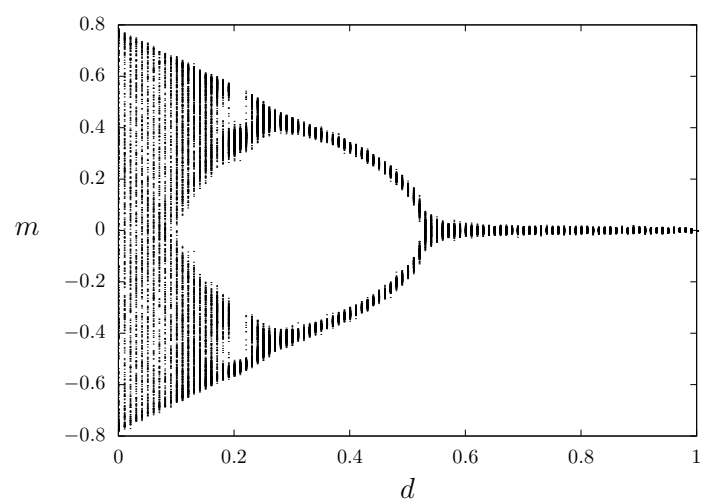

Fig. 7. Bifurcations as a function of the dilution $d$. Here, $J=-10, W=20$ $p=1,500$ points, $N=10,000$, transient of 1000 steps, three initial conditions $\left(m_{0}=0.2,0.5,0.8\right)$. The gap near $d=0.2$ is due to a pitchfork bifurcation not captured by the set of initial conditions.

\section{Conclusions}

We investigated the phase transitions of a nonlinear, parallel version of the Ising model, characterized by a linear coupling $J<0$ and a nonlinear one $W>0$.

The mean-field approximation shows chaotic oscillations, by changing the linear coupling $J$ or the connectivity $K$. We show that there is a form of mapping among the parameters $J, W$ and $K$, so that only two of them are independent.

The spatial model exhibits bifurcations in the average magnetization, similar to what is seen in the mean-field approximation, induced by the change of the topology, after rewiring short-range to long-range connections. Indeed, this is a consequence of the small-world effect [12], for which a sufficiently large number of long-range connections make the system behave as the mean-field approximation. In the spatial model, the periodic and chaotic oscillations of the magnetization imply a certain degree of synchronization of the spins, induced by long-range couplings.

We have also shown that similar bifurcations may be induced in the randomly connected model by changing the parameters $J$, the connectivity $K$ and also the asynchronism factor $d$.

This model may be useful in modelling collective effects in systems that take decisions based on those coming from peers. In particular, we think that this situation may be important for high-frequency algorithmic trading. In this case, due to the necessity of performing actions in the smallest possible time, all operations are carried out by algorithms, in an almost parallel way. The contrarian attitude is a well-known practice, coupled with the necessity 
of following the majority in the case of consensus (see the discussion in Ref. [4]). Our model shows that in this case, chaotic oscillations may arise, corresponding, in the finance language, to crashes and crisis. As shown, these effects may be mitigated by the asynchronism and by local interactions.

This work was partially supported by project PAPIIT-DGAPA-UNAM IN109213. F.B. acknowledges partial financial support from the European Commission (FP7-ICT-2011-7) Proposal No. 288021 EINS - Network of Excellence in Internet Science and European Commission (FP7-ICT-201310) Proposal No. 611299 SciCafe 2.0.

\section{REFERENCES}

[1] C. Castellano, S. Fortunato, V. Loreto, Rev. Mod. Phys. 81, 591 (2009).

[2] J. Touboul, arXiv:1410.8001 [cond-mat.dis-nn].

[3] S.E. Asch, Effects of Group Pressure on the Modification and Distortion of Judgments, in: Groups, Leadership and Men, H. Guetzkow (Ed.), Carnegie Press, Pittsburgh PA 1951, pp. 177-190; Social Psychology, Prentice Hall, Englewood Cliffs NJ, 1952; Psychol. Monogr.:Gen. Appl. 70, 1 (1956).

[4] F. Bagnoli, R. Rechtman, Phys. Rev. E 88, 062914 (2013).

[5] F. Bagnoli, R. Rechtman, Phys. Rev. E 92, 042913 (2015).

[6] W.B. Arthur, Am. Econ. Rev. 84, 406 (1994); D. Challet, Y.-C. Zhang, Physica A 246, 407 (1997); D. Challet, M. Marsili, Y.-C. Zhang, Minority Games, Oxford University Press, 2005; A.C.C. Coolen, The Mathematical Theory of Minority Games, Oxford University Press, 2005.

[7] P.S. Dodds, K.D. Harris, C.M. Danforth, Phys. Rev. Lett. 110, 158701 (2013).

[8] K.D. Harris, C.M. Danforth, P.S. Dodds, Phys. Rev. E 88, 022816 (2013).

[9] F. Bagnoli, T. Matteuzzi, R. Rechtman, Acta. Phys. Pol. B Proc. Suppl. 9, 9999 (2016), this issue.

[10] B. Derrida, Dynamical Phase Transitions in Spin Models and Automata, in:

H. Beijeren (Ed.), Fundamental Problems in Statistical Mechanics VII, p. 276, Elsevier, 1990.

[11] A.U. Neumann, B. Derrida, J. Physique 49, 1647 (1988).

[12] D.J. Watts, S.H. Strogatz, Nature 393, 409 (1998).

[13] E. Ott, Chaos in Dynamical Systems, Cambridge University Press, Cambridge UK, 2002. 